\title{
First trough level of infliximab at week 2 predicts future outcomes of induction therapy in ulcerative colitis-results from a multicenter prospective randomized controlled trial and its post hoc analysis
}

\author{
Taku Kobayashi ${ }^{1} \cdot$ Yasuo Suzuki $^{2} \cdot$ Satoshi Motoya $^{3} \cdot$ Fumihito Hirai $^{4}$ • \\ Haruhiko Ogata ${ }^{5}$ Hiroaki Ito ${ }^{6} \cdot$ Noriko Sato $^{7} \cdot$ Kunihiko Ozaki $^{7}$. \\ Mamoru Watanabe ${ }^{8}$ Toshifumi Hibi ${ }^{1}$
}

Received: 28 April 2015/ Accepted: 25 June 2015/Published online: 11 July 2015

(C) The Author(s) 2015. This article is published with open access at Springerlink.com

\begin{abstract}
Background Infliximab (IFX) is one of the treatments of choice for corticosteroid-refractory and corticosteroid-dependent ulcerative colitis (UC). A high serum trough level of IFX (TL) is reported to be associated with sustained efficacy during maintenance treatment. As part of a phase 3 randomized controlled trial of IFX in UC, we assessed the predictive value of the first TL at week 2 for short- and long-term response.

Methods Patients received intravenous IFX $5 \mathrm{mg} / \mathrm{kg}$ or placebo at weeks 0,2 , and 6 . Patients with evidence of a response by week 8 continued treatment at weeks 14 and 22. TL was measured by enzyme-linked immunosorbent
\end{abstract}

Toshifumi Hibi

thibi@insti.kitasato-u.ac.jp

1 Center for Advanced IBD Research and Treatment, Kitasato University Kitasato Institute Hospital, 5-9-1 Shirokane, Minato-ku, Tokyo 108-8642, Japan

2 Department of Internal Medicine, Toho University Sakura Medical Center, Sakura, Japan

3 Inflammatory Bowel Diseases Center, Sapporo-kosei General Hospital, Sapporo, Japan

4 Department of Gastroenterology, Fukuoka University Chikushi Hospital, Chikushino, Japan

5 Center for Diagnostic and Therapeutic Endoscopy, Keio University, School of Medicine, Tokyo, Japan

6 Digestive Disease Center, Kitano Hospital, The Tazuke Kofukai Medical Research Institute, Osaka, Japan

7 Mitsubishi Tanabe Pharma Corporation, Osaka, Japan

8 Department of Gastroenterology and Hepatology, Tokyo Medical and Dental University, Tokyo, Japan assay. Post hoc analysis was then performed for TL and clinical outcomes.

Results Clinical response rate at week 8, the primary end point, was significantly higher in the IFX group than placebo $(p=0.005)$. The incidence of adverse events between groups was similar. Week 2 TL was significantly associated with a 14-week clinical activity index (CAI) remission. In multiple logistic regression analysis, the week 2 TL-to-CAI ratio (TL/CAI, odds ratio 8.07; $95 \%$ confidence interval 2.84-27.07, $p<0.001$ ) was an independent factor correlating with 14-week CAI remission. The week 2 TL and TL/CAI were also significantly associated with 30-week mucosal healing.

Conclusions IFX was confirmed to be effective and safe in this population. Our results suggest that the first TL at week 2 , in combination with clinical evaluation, is useful for predicting both short- and long-term outcomes, allowing an earlier decision between continuing IFX or switching to other options.

Keywords Ulcerative colitis - Infliximab - Therapeutic drug monitoring $\cdot$ Primary response $\cdot$ Mucosal healing

\section{Introduction}

Ulcerative colitis (UC) and Crohn's disease (CD) are two major forms of chronic inflammatory bowel disease [1]. Although their etiology remains unknown, immunological, genetic, and environmental factors are considered to play important roles in their pathogenesis [2,3]. Tumor necrosis factor-alpha (TNF- $\alpha$ ) is one of the cytokines reported to be increased in both UC and CD [4, 5], and TNF- $\alpha$ blockade has emerged as an effective therapeutic strategy for both diseases. The first use of the anti-TNF- $\alpha$ antibody 
infliximab (IFX) for human inflammatory bowel disease was reported in a pediatric patient with severe CD in 1997 [6]. Efficacy in $\mathrm{CD}$ was confirmed in the subsequent ACCENT I and II randomized controlled trials [7]. Efficacy for UC was studied in the 54-week ACT 1 and 30-week ACT 2 trials, in which the IFX groups showed statistically significant increases in the proportion of patients with a clinical response, clinical remission, and mucosal healing $(\mathrm{MH})$ compared with placebo [8].

Intravenous corticosteroid administration remains the first-line therapy in UC patients with acute moderate-tosevere disease and is reported to be effective in more than half of them [9]. However, a certain proportion of patients is refractory to corticosteroid treatment. Moreover, even among initial responders, approximately one-third of patients cannot withdraw from corticosteroids without a symptom flare, or experience symptoms after steroid discontinuation, and are therefore considered steroid-dependent. While IFX is a treatment of choice in steroid-refractory and steroid-dependent patients, other options that can be used for these patients as a rescue therapy include anti-TNF$\alpha$ monoclonal antibodies (adalimumab and golimumab), calcineurin inhibitors (tacrolimus and cyclosporine), anti$\alpha_{4} \beta_{7}$ integrin (vedolizumab), and cytapheresis [10, 11]. No consensus on a strategy for corticosteroid-refractory or corticosteroid-dependent moderate-to-severe UC patients has been reached, since evidence directly comparing these options does not exist, except for a head-to-head comparison showing no difference between IFX and cyclosporine [12]. In addition, no definitive predictive factor has been reported to help choose a given treatment option.

Monitoring serum concentrations of IFX has been considered useful in evaluating the mechanism of loss of response to IFX. Afif et al. reported that low drug levels drawn 4 or 8 weeks after a scheduled infusion every 8 weeks predict loss of response after initial success with IFX [13]. A therapeutic algorithm for patients with secondary failure based on serum IFX trough levels (TLs) and the development of antibodies (ATIs) against IFX has been suggested to help determine an appropriate treatment intervention [13, 14].

Acute severe flares are one of the most critical features of UC. They may require hospitalization and surgery. Because decisions on whether a treatment should be continued or switched to the next option are often made with a lack of data about possible outcomes, and may result in necessary surgery being delayed, the earliest possible evaluation of the response to treatment is crucial. In clinical practice, judging short- and long-term efficacy soon after the initial infusion of IFX is always challenging: some patients gradually respond over time after multiple infusions, whereas others demonstrate a transient response followed by a subsequent loss of efficacy. Thus, the availability of an early and accurate predictor of outcome would be of particular value.

Here, as part of a phase 3 trial of IFX efficacy and safety in UC, we examined the accuracy of TL measurements as a predictive factor of therapeutic response early in IFX treatment.

\section{Methods}

\section{Patients}

This randomized, double-blind, placebo-controlled study (Japic CTI-060298) was conducted between July 2006 and December 2008 among 208 patients at 67 medical institutions in Japan. The protocol was approved by the institutional review board at each medical institution. All patients gave written informed consent. The study was conducted in accordance with the Declaration of Helsinki and Good Clinical Practice.

All eligible patients had an established diagnosis of UC and had been screened for tuberculosis by the skin test and chest radiography. Endoscopy was performed during screening to confirm disease activity. Eligible patients had no evidence of tuberculosis and had active UC with a Mayo score [15] of 6-12 points and an endoscopic subscore of $\geq 2$. Documentation of one or more previous treatment failures was required at enrollment, namely, we enrolled patients with no response to immunomodulators (azathioprine and 6-mercaptopurine) that were started at least 12 weeks before enrollment and maintained at a stable dose from at least 4 weeks before enrollment or that were administered for at least 12 weeks within the preceding 5 years; patients who could not tolerate immunomodulators within the preceding 5 years; patients who had had no response to oral corticosteroids started at least 2 weeks before enrollment at $20 \mathrm{mg} /$ day or more and maintained at a stable dose (corticosteroid-refractory); and patients who showed no response to corticosteroids (equivalent prednisolone $40 \mathrm{mg} /$ day or more, for at least 2 weeks orally, or for at least a week intravenously), worsening of UC with tapering of corticosteroids (corticosteroid-dependent), or corticosteroid intolerance within the previous 18 months.

We excluded patients with a history of recent bowel surgery; bowel complications such as stricture, fistula, or dysplasia; or treatment with other biologics, methotrexate, calcineurin inhibitors, or cytapheresis within the previous 18 months because of the possible influence of these treatments on the efficacy of IFX. Patients with serious medical conditions such as chronic heart failure or latent infectious diseases (hepatitis $\mathrm{C}$, human immunodeficiency virus infection, or any other chronic infectious disease) were also excluded. 


\section{Study design}

Eligible patients were randomly assigned in a 1:1 ratio to receive an intravenous infusion of IFX at a dose of $5 \mathrm{mg} / \mathrm{kg}$ or placebo at weeks 0,2 , and 6 (Fig. 1a). Randomization was performed centrally with the use of computer-generated randomization schedules stratified according to the investigational site and concomitant use or nonuse of corticosteroids (prednisolone equivalent of $0,<20$, or $\geq 20 \mathrm{mg}$ /day). Patients with a significantly lower Mayo score at week 8 (defined as a decrease in the total Mayo baseline score of at least 3 points and at least $30 \%$, with an accompanying decrease in the rectal bleeding subscore of at least 1 point or an absolute subscore of 0 or 1 , i.e., 8 -week responders) then received IFX or placebo at weeks 14 and 22. Conversely, 8-week non-responders were discontinued from IFX or placebo treatment. Eight-week responders were followed through week 38. Eight-week non-responders and other patients who discontinued treatment with IFX or placebo were followed until 16 weeks after the last administration.

Permitted concomitant medications for UC included corticosteroids (started at least 2 weeks before sigmoidoscopy at enrollment and maintained at a stable dose), oral aminosalicylates (started at least 3 weeks before enrollment and maintained at a stable dose), azathioprine and 6-mercaptopurine (started at least 12 weeks before enrollment and maintained at a stable dose from at least 4 weeks before enrollment). Doses of concomitant medications remained constant throughout the study except for corticosteroids, which were tapered by $5 \mathrm{mg} /$ week after week 8 until a dose of $20 \mathrm{mg} /$ day was reached and then by $2.5 \mathrm{mg} /$ week.

\section{End points}

The primary end point was a clinical response at week 8 . Secondary end points were clinical remission or $\mathrm{MH}$
Fig. 1 Study schema (a), patient disposition (b). IFX infliximab; $U C$ ulcerative colitis; IC informed consent

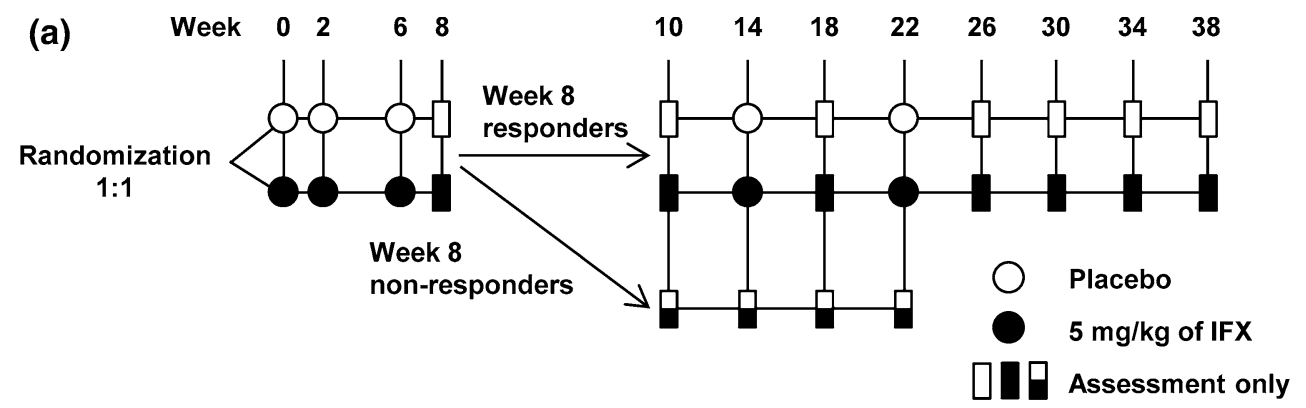

(b)

208 patients randomly assigned to treatment at week 0

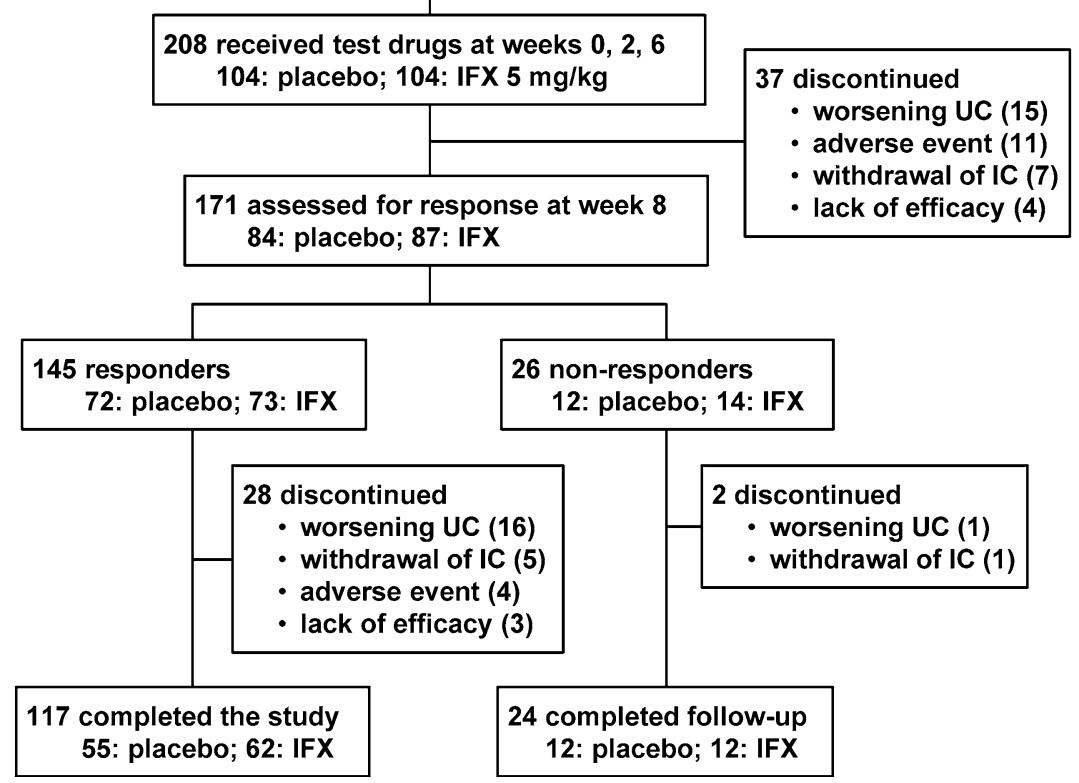


response at week 8 as well as clinical response or clinical remission at week 30. The Mayo score was determined at weeks 0,8 , and 30. Clinical remission was defined as a total Mayo score of 2 points or lower, with no individual subscore exceeding 1 point. MH was defined as an endoscopic subscore of 0 or 1 . The clinical activity index (CAI) [16] was determined at all visits. CAI remission was defined as a CAI score of 4 or lower.

TL at weeks $0,2,6$, and every 8 weeks thereafter and ATI at weeks 0, 14, and 30 were measured using an enzyme-linked immunosorbent assay [17] (Mitsubishi Tanabe Pharma Corp., Osaka, Japan).

\section{Statistical analysis}

Efficacy was assessed in the full analysis set. Patients who took prohibited medication because of worsening UC (lack of efficacy or loss of response to the study medication), who discontinued the study medication because of worsening UC, including 8-week non-responders, or who underwent colectomy or colostomy were not considered to have had a clinical response, clinical remission, or $\mathrm{MH}$, and their post-procedure CAI score was used as the baseline value from the time of the procedure onward. For other patients who withdrew prematurely, the last observation was carried forward. Statistical tests were two-sided, with $p \leq 0.05$ considered to indicate statistical significance.

Demographic and baseline characteristics were compared using Fisher's exact test, Student's $t$ test, or the Wilcoxon rank test. Proportions of patients with a clinical response, clinical remission, and $\mathrm{MH}$ were compared using logistic regression analysis [explanatory variables: treatment group, corticosteroid use (except for analysis in severe cases)].

\section{Post hoc analysis}

We conducted post hoc analysis in 82 patients who were treated with IFX at weeks 0,2 , and 6 and evaluated until week 14. The ratio of TL to CAI (TL/CAI) was calculated using the CAI value plus 1 , because CAI scores include 0 . Between-group differences in the TL and CAI remission rates were calculated with the Wilcoxon rank test and Fisher's exact test, respectively. Receiver-operating characteristic (ROC) curve analysis was performed to assess the performance of the week 2 TL or TL/CAI for predicting CAI remission at week 14 . Predictive factors in baseline characteristics as well as clinical and laboratory parameters identifying patients with CAI remission at week 14 were analyzed with logistic regression analysis. The cutoff values for age, body weight, TL, and TL/CAI used median values, while those for other end points used their reference values. Multiple logistic regression analysis was performed to identify independent predictors of 14-week CAI remission. The explanatory variables used in these multiple analyses were sex, age $<39.5$ years, weight $<56.0 \mathrm{~kg}$, smoker, colonic area involved, oral corticosteroid use, oral 5-aminosalicylate use, immunomodulator use, corticosteroid-refractory disease, corticosteroid-dependent disease, CAI remission at week 2 , the week $2 \mathrm{TL}$ (first TL) $\geq$ the median value, and the first $\mathrm{TL} / \mathrm{CAI} \geq$ the median value. Variables were chosen using stepwise backward selection to minimize the Bayesian information criterion.

Mitsubishi Tanabe Pharma Corp. sponsored this clinical trial and was responsible for the collection and analysis of data.

\section{Results}

\section{Clinical outcome}

Patient disposition is shown in Fig. 1b. There were no significant differences in backgrounds between the IFX and placebo groups (Table 1). At week 8, the clinical response rate, the primary end point, was significantly higher in patients who received IFX than in those who received placebo ( $p=0.005$; Table 2$)$. The clinical remission rate showed marginal significance $(p=0.054)$, whereas $\mathrm{MH}$ was significantly more frequent with IFX than placebo $(p=0.006)$. Results of clinical response, clinical remission, and $\mathrm{MH}$ at week 30 are shown in Table 2. Response rate at week 8 in acute severe cases ( $n=18$, Mayo score $\geq 11$ ) was similar to that in the entire study population, although it did not reach statistical significance because of the limited number of patients [54.5\% (6/11) in the IFX group versus $28.6 \%(2 / 7)$ in the placebo group, $p=0.287$ ].

Since this study was conducted as part of a nationwide phase 3 trial in Japan, safety profiles were also carefully calculated for all patients. There was no difference in the incidence of any adverse events, including serious infections and infusion reactions, during the short-term as well as the entire follow-up period in this trial (Table 3 ).

\section{Clinical course of patients treated with IFX}

We next assessed the clinical course of 82 patients who received the initial three infusions at weeks 0,2 , and 6 and evaluated them until week 14 to focus on the initial response to induction therapy with IFX, using CAI determined at all visits. Among these 82 patients, 37 (45\%) achieved CAI remission at week 2, but $8(22 \%)$ lost their response by week 14 (Fig. 2a). In contrast, 17 (38\%) patients who were not in CAI remission at week 2 improved and achieved CAI remission at week 14. Interestingly, most patients who achieved CAI remission 
Table 1 Baseline characteristics of patients

\begin{tabular}{|c|c|c|c|}
\hline Characteristic & Placebo $(n=104)$ & $\operatorname{IFX}^{\mathrm{a}}(n=104)$ & $p$ \\
\hline Male sex $n(\%)$ & $67(64.4)$ & $66(63.5)$ & $1.000^{\mathrm{c}}$ \\
\hline Age (years) & $37.8 \pm 12.9$ & $40.0 \pm 12.7$ & $0.220^{\mathrm{d}}$ \\
\hline Median $(\mathrm{IQR})^{\mathrm{b}}$ & $36.0(28.0,47.0)$ & $39.5(29.5,49.0)$ & \\
\hline Weight $(\mathrm{kg})$ & $60.3 \pm 11.6$ & $57.6 \pm 12.7$ & $0.117^{\mathrm{d}}$ \\
\hline Median (IQR) & $59.0(52.0,69.0)$ & $56.0(49.0,64.0)$ & \\
\hline Smoking $n(\%)$ & $6(5.8)$ & $9(8.7)$ & $0.593^{\mathrm{c}}$ \\
\hline Duration of disease (years) & $7.1 \pm 6.6$ & $8.1 \pm 7.2$ & \\
\hline Median (IQR) & $4.7(2.5,9.2)$ & $6.5(3.5,11.0)$ & $0.133^{\mathrm{e}}$ \\
\hline \multicolumn{4}{|l|}{ Colonic area involved } \\
\hline Left side $n(\%)$ & $20(19.2)$ & $21(20.2)$ & $1.000^{\mathrm{c}}$ \\
\hline Extensive $n(\%)$ & $84(80.8)$ & $83(79.8)$ & \\
\hline \multicolumn{4}{|l|}{ Concomitant medications $n(\%)$} \\
\hline Corticosteroids (oral) & $69(66.3)$ & $68(65.4)$ & $1.000^{\mathrm{c}}$ \\
\hline 5-Aminosalicylates (oral) & $70(67.3)$ & $77(74.0)$ & $0.361^{\mathrm{c}}$ \\
\hline Immunomodulators & $49(47.1)$ & $50(48.1)$ & $1.000^{\mathrm{c}}$ \\
\hline Azathioprine & $34(32.7)$ & $38(36.5)$ & $0.662^{\mathrm{c}}$ \\
\hline 6-Mercaptopurine & $15(14.4)$ & $12(11.5)$ & $0.681^{\mathrm{c}}$ \\
\hline Corticosteroid-refractory disease $n(\%)$ & $20(19.2)$ & $20(19.2)$ & $1.000^{\mathrm{c}}$ \\
\hline Corticosteroid-dependent disease $n(\%)$ & $52(50.0)$ & $44(42.3)$ & $0.330^{\mathrm{c}}$ \\
\hline Mayo score & $8.5 \pm 1.4$ & $8.6 \pm 1.4$ & $0.435^{\mathrm{c}}$ \\
\hline Median (IQR) & $9.0(7.0,9.5)$ & $9.0(8.0,9.0)$ & \\
\hline Clinical activity index ${ }^{\mathrm{f}}$ & $8.2 \pm 2.4$ & $8.4 \pm 2.6$ & $0.502^{\mathrm{d}}$ \\
\hline Median (IQR) & $8.0(6.0,10.0)$ & $8.0(7.0,10.0)$ & \\
\hline C-reactive protein $\mathrm{mg} / \mathrm{dl}$ & $0.7 \pm 1.1$ & $1.0 \pm 1.5$ & \\
\hline Median (IQR) & $0.4(0.1,0.9)$ & $0.5(0.1,1.0)$ & $0.092^{\mathrm{e}}$ \\
\hline Albumin $\mathrm{g} / \mathrm{dl}$ & $3.9 \pm 0.4$ & $3.9 \pm 0.4$ & \\
\hline Median (IQR) & $3.9(3.7,4.2)$ & $3.9(3.7,4.1)$ & $0.400^{\mathrm{e}}$ \\
\hline
\end{tabular}

a IFX infliximab

${ }^{b}$ IQR interquartile range

c Fisher's exact test

d Student's $t$-test

e Wilcoxon rank test

${ }^{\mathrm{f}}$ Placebo, $n=104$, infliximab, $n=103$

Data are shown as the mean \pm SD except where indicated otherwise at week 14 remained in remission until week 30 , more frequently than the week 8 CAI remitters. Week 14 therefore appeared to be an appropriate time point to define the primary clinical response for the following analyses. TL was significantly higher in patients who were in CAI remission than in those who were not at each time point after the second infusion at week 6 (Fig. 2b).

\section{Assessment of TL}

Early prediction of clinical efficacy is especially important for the treatment of acutely ill patients. Therefore, we conducted a post hoc analysis to examine the potential usefulness of TL at the early time point for prediction of future outcome.

Interestingly, an association between week $2 \mathrm{TL}$ and clinical efficacy showed a tendency to be stronger at the later time point (Fig. 3a) [the optimal cutoff value for predicting 14-week CAI remission: $21.3 \mu \mathrm{g} / \mathrm{ml}$; 0.61 sensitivity, 0.69 specificity, 0.72 positive predictive value, and 0.58 negative predictive value; area under the curve: 0.65 , $95 \%$ confidence interval (CI): 0.52-0.77]. The correlation of TL with $\mathrm{MH}$ was also evaluated, since $\mathrm{MH}$ is considered an important predictive factor for long-term outcome. Similar to clinical efficacy, the first TL showed a significant correlation with $\mathrm{MH}$ at week 30 but not at week 8 (Fig. 3b). 
Table 2 Summary of efficacy results

\begin{tabular}{|c|c|c|c|}
\hline Characteristics & Placebo $(n=104)$ & $\operatorname{IFX}^{\mathrm{a}}(n=104)$ & $p^{\mathrm{b}}$ \\
\hline \multicolumn{4}{|c|}{ Clinical response $n(\%)$} \\
\hline Week 8 & 37 (35.6) & $57(54.8)$ & 0.005 \\
\hline Week 30 & 33 (31.7) & $48(46.2)$ & 0.033 \\
\hline \multicolumn{4}{|c|}{ Clinical remission $n(\%)$} \\
\hline Week 8 & $11(10.6)$ & $21(20.2)$ & 0.054 \\
\hline Week 30 & $17(16.3)$ & $22(21.2)$ & 0.373 \\
\hline \multicolumn{4}{|c|}{ Mucosal healing $n(\%)$} \\
\hline Week 8 & 29 (27.9) & $48(46.2)$ & 0.006 \\
\hline Week 30 & $30(28.8)$ & $43(41.3)$ & 0.057 \\
\hline
\end{tabular}

${ }^{\text {a }}$ IFX, infliximab

b Logistic regression analysis (explanatory variable: treatment group, corticosteroid use)

\section{Usefulness of the first $T L$ in predicting clinical outcome}

We screened for the predictive values of multiple clinical parameters, including background and 2-week clinical parameters. Results showed that there was no patient background factor, including the severity and laboratory data, that demonstrated a significant association with clinical outcome at week 14 (Table 4). However, CAI remission at 2 weeks was significantly associated with the 14-week CAI remission, in addition to the first TL. This suggests that patients already in remission at week 2 are likely to remain in remission at week 14 . However, this is not useful in identifying patients who gain/lose their response to IFX after week 2 . We therefore evaluated the first TL/CAI and found that this ratio also correlated with 14-week CAI remission (Table 4) (the optimal cutoff value for predicting 14-week CAI remission: 4.3; 0.61 sensitivity, 0.81 specificity, 0.80 positive predictive value, and
0.62 negative predictive value; area under the curve: 0.76 , $95 \%$ CI: 0.65-0.86). Accordingly, although it did not reach statistical significance because of the limited sample size, the week 14 CAI remission rate in 2-week nonremitters (subgroup of delayed response) with the first TL/ CAI of $\geq 4.3$ tended to be higher than in those with the first TL/CAI $<4.3(50 \%$ versus $36 \%)$. Furthermore, the week $14 \mathrm{CAI}$ non-remission rate in 2-week remitters (subgroup of loss of response) with the first TL/CAI of $\geq 4.3$ tended to be lower than in those with the first TL/CAI $<4.3(14 \%$ versus $44 \%$ ). In multiple logistic analysis, the first TL/CAI (odds ratio 8.07, $95 \%$ confidence interval 2.84-27.07, $p<0.001$ ) and 5-aminosalicylate use (odds ratio 3.98, $95 \%$ confidence interval $1.29-14.26, p=0.016)$ were identified as independent factors that correlated with 14-week CAI remission. The first TL/CAI was significantly associated with 30 -week $\mathrm{MH}$ as well as with 14-week CAI remission (Fig. 3c, d).

\section{Discussion}

We assessed the predictive value of the first TL at week 2 for clinical outcome as part of a phase 3 randomized controlled trial of IFX in UC. Results showed the utility of the week 2 first TL as a predictive factor for both short- and long-term outcomes in these patients.

Previous studies reported that low serum albumin, absence of concomitant immunomodulator use, and severe disease were associated with poor response to IFX [18-21]. However, in our study population, neither these nor any other baseline characteristics were associated with clinical outcome, suggesting that patient background, including severity and laboratory data, is not useful in predicting clinical outcome before the start of therapy. Therefore,

Table 3 Safety profiles

\begin{tabular}{|c|c|c|c|c|}
\hline & \multicolumn{2}{|l|}{ By week 14} & \multicolumn{2}{|l|}{ Entire period } \\
\hline & Placebo $(n=104)$ & $\operatorname{IFX}^{\mathrm{a}}(n=104)$ & Placebo $(n=104)$ & $\operatorname{IFX}(n=104)$ \\
\hline Follow-up days, mean (range) & $98.3(92-106)$ & $98.1(66-106)$ & $214.1(111-274)$ & $223.2(66-274)$ \\
\hline Mean doses $n$ & 2.7 & 2.9 & 3.9 & 4.2 \\
\hline Any adverse event & $86(82.7)$ & $85(81.7)$ & $94(90.4)$ & $100(96.2)$ \\
\hline Infections & $35(33.7)$ & $33(31.7)$ & $51(49.0)$ & $62(59.6)$ \\
\hline Infusion reactions & $9(8.7)$ & $11(10.6)$ & $11(10.6)$ & $16(15.4)$ \\
\hline Adverse events leading to discontinuation of study drug & $8(7.7)$ & $5(4.8)$ & $8(7.7)$ & $7(6.7)$ \\
\hline Any serious adverse event & $13(12.5)$ & $9(8.7)$ & $19(18.3)$ & $18(17.3)$ \\
\hline Worsening ulcerative colitis & $11(10.6)$ & $8(7.7)$ & $18(17.3)$ & $16(15.4)$ \\
\hline Serious infections & $2(1.9)$ & $1(1.0)$ & $2(1.9)$ & $1(1.0)$ \\
\hline Serious infusion reactions & $0(0.0)$ & $0(0.0)$ & $0(0.0)$ & $0(0.0)$ \\
\hline
\end{tabular}

${ }^{a}$ IFX, infliximab

Data are shown as the number of patients (\%) except where indicated otherwise 
Fig. 2 Clinical course and TL of patients treated with IFX. a Clinical course of patients who achieved CAI remission. b TL according to clinical efficacy. Results are shown as median (interquartile range). Statistical differences were calculated with the Wilcoxon rank test. $T L$ serum infliximab (IFX) trough level; CAI clinical activity index (a).

Clinical course at weeks 2,14 , and 30

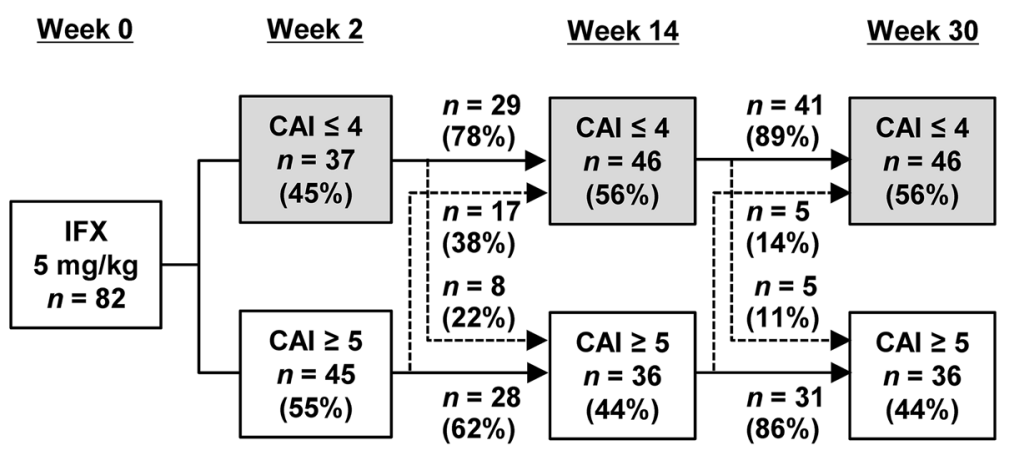

- Clinical course at weeks 2, 8, and 30
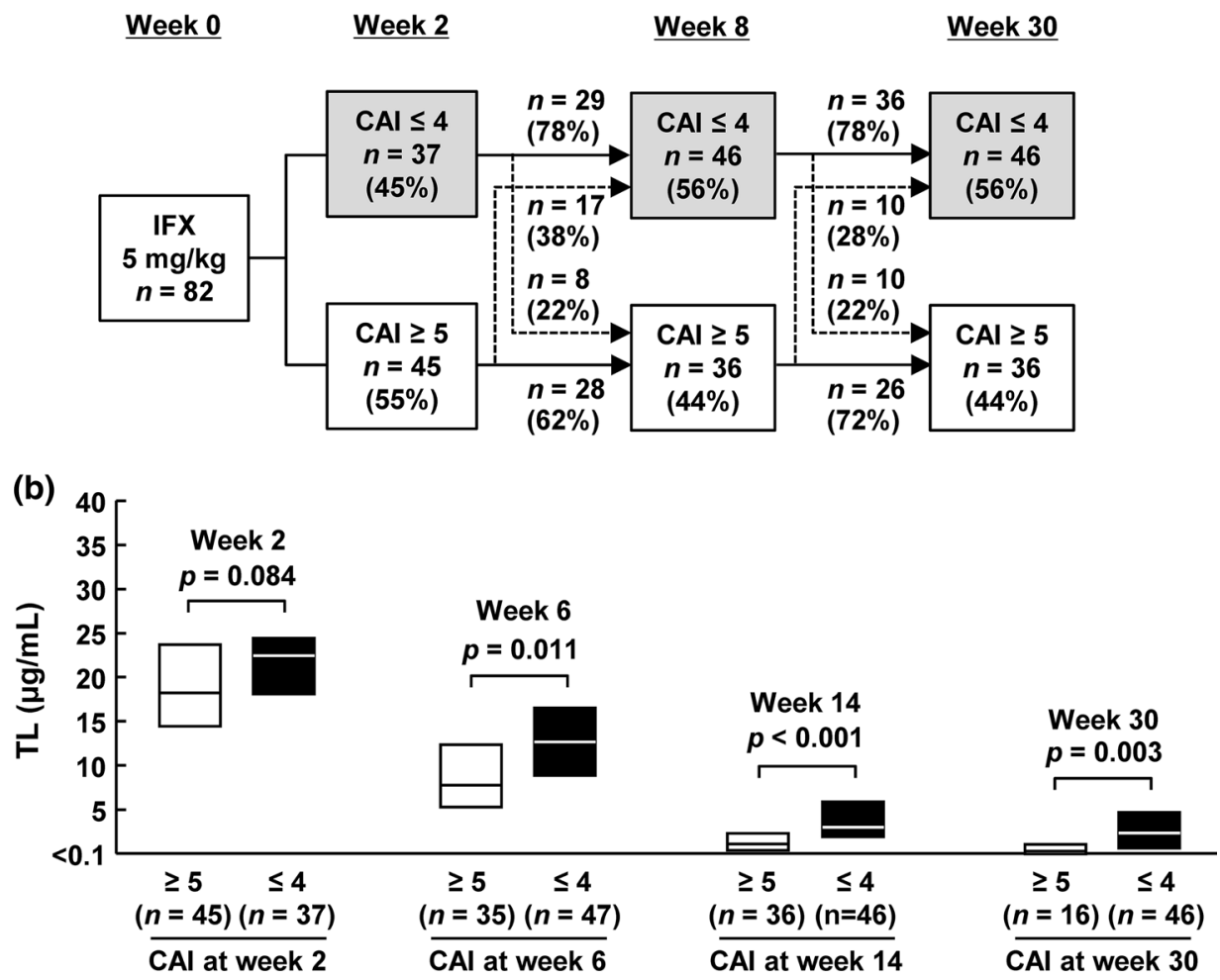

early detection of response to IFX is critical for the management of UC, especially in acute severe patients (e.g., Mayo score $\geq 11$ or $\mathrm{CAI} \geq 12$ ), whose clinical outcome was comparable with that in the moderately active patients. It has been reported that TL is associated with the clinical status at each time point in patients under maintenance therapy [22, 23], but its predictive role for future outcomes during induction therapy has not been studied. Therefore, we hypothesized that the first TL may be useful and found that the first TL significantly correlates with 14-week primary response. Interestingly, the correlation between the first TL and clinical outcome was seen even at week 30, suggesting that the 30 -week clinical efficacy can be predicted after as little as 2 weeks. Furthermore, we also found that the first TL correlated with the achievement of $\mathrm{MH}$ at 30 weeks. Post hoc analysis was conducted in 82 patients who received treatment at weeks 0,2 , and 6 and were evaluated until week 14 . However, even after including the 11 patients who discontinued the therapy because of worsening UC or lack of efficacy by week 14, the first TL showed significant correlations with 14-week remission and 30-week MH (data not shown). Although we did not conduct a long-term follow-up in this study, our results suggest that the first TL may predict not only the short-term, but also long-term outcome of subsequent IFX maintenance therapy, since $\mathrm{MH}$ is reported to be associated 
Fig. 3 TL at week 2 according to clinical efficacy at weeks 2 , 6,14 , and $30(\mathbf{a}), \mathrm{MH}$ at weeks 8 and 30 (b), 2-week TL/CAI according to 14-week CAI remission (c), and 30-week MH (d). Results are shown as median (solid line within box or bar) and interquartile range (upper and lower box boundaries). Statistical differences were calculated with the Wilcoxon rank test. $T L$ serum infliximab trough level; $C A I$ clinical activity index; $M H$ mucosal healing

(a)

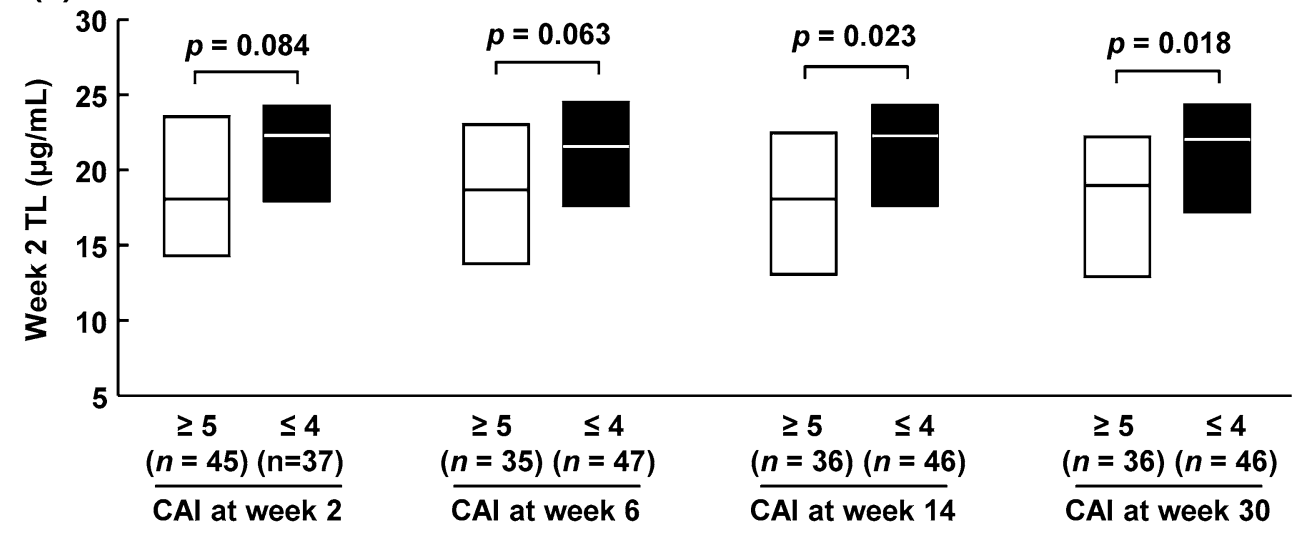

(b)

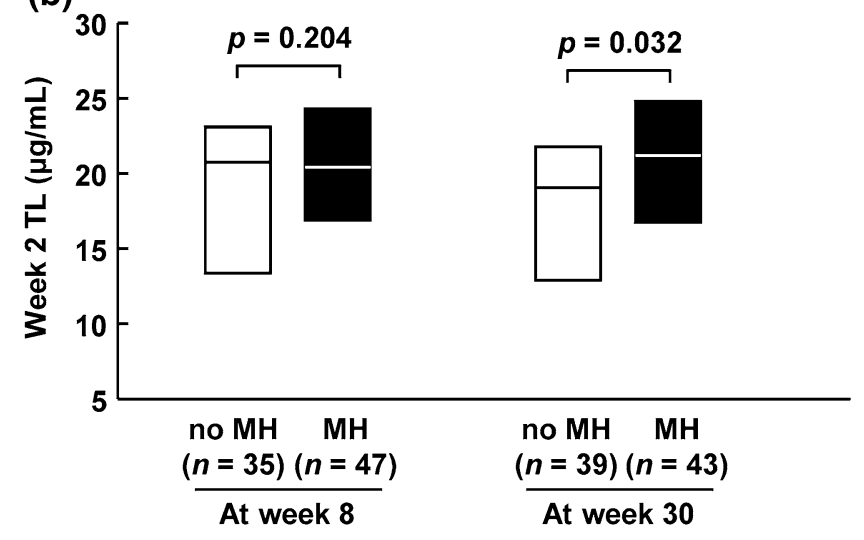

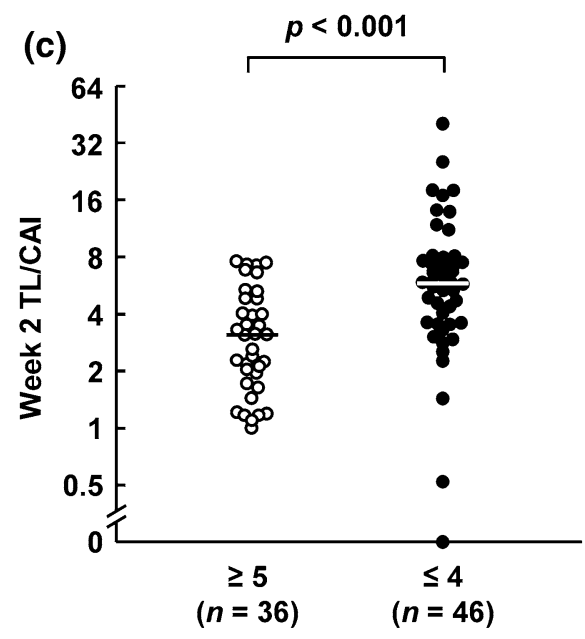

CAl at week 14

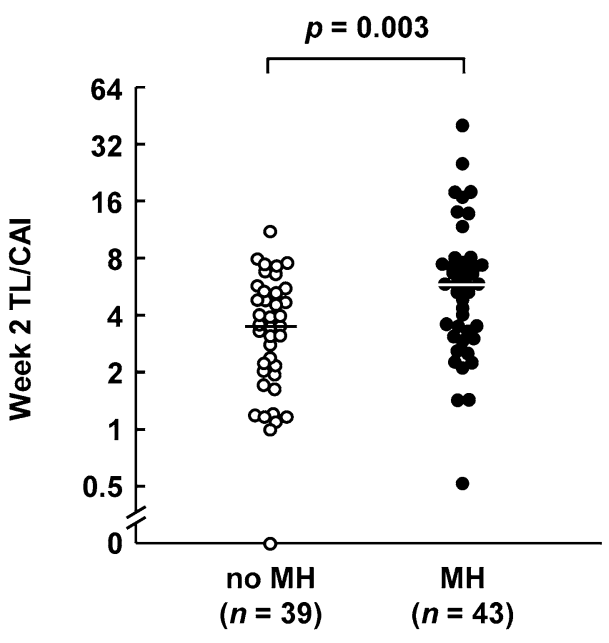

At week 30 with a favorable long-term outcome [24]. This may be of major benefit in helping both patients and physicians to decide whether or not to continue IFX after 2 weeks.

On further investigation, we found that the first TL/CAI is an independent predictive factor for early clinical efficacy and significantly associated with subsequent $\mathrm{MH}$. This seems clinically relevant since the TL/CAI may reflect the drug level required to treat residual inflammation in each patient. Therefore, TL measurement in combination with re-evaluation of clinical severity at week 2 appears to be a reasonable strategy for predicting both short- and long-term treatment outcomes with IFX while still in the initial stage of treatment.

One clinical question arising from our results is whether early dose escalation or elective switching could improve the outcome in patients whose first TL or TL/CAI appears 
Table 4 Predictive factors associated with 14-week CAI remission (univariate analysis)

\begin{tabular}{|c|c|c|c|}
\hline \multirow[t]{2}{*}{ Characteristic } & \multicolumn{3}{|c|}{ 14-Week $\mathrm{CAI}^{\mathrm{a}}$ remission } \\
\hline & Odds ratio & $95 \% \mathrm{CI}^{\mathrm{b}}$ & $p$ \\
\hline \multicolumn{4}{|l|}{ Baseline } \\
\hline Male sex & 1.06 & $0.42,2.64$ & 0.901 \\
\hline Age $<39.5$ years & 0.62 & $0.25,1.48$ & 0.277 \\
\hline Weight $<56.0 \mathrm{~kg}$ & 0.86 & $0.36,2.07$ & 0.735 \\
\hline Smoker & 2.07 & $0.42,15.11$ & 0.383 \\
\hline \multicolumn{4}{|l|}{ Colonic area involved } \\
\hline Extensive & 0.40 & $0.10,1.29$ & 0.129 \\
\hline \multicolumn{4}{|l|}{ Concomitant medications } \\
\hline Corticosteroids (oral) & 1.27 & $0.49,3.28$ & 0.621 \\
\hline 5-Aminosalicylates (oral) & 2.02 & $0.79,5.35$ & 0.144 \\
\hline Immunomodulators & 0.67 & $0.28,1.61$ & 0.373 \\
\hline Azathioprine & 0.68 & $0.27,1.68$ & 0.399 \\
\hline 6-Mercaptopurine & 0.93 & $0.26,3.49$ & 0.911 \\
\hline Corticosteroid-refractory disease & 1.95 & $0.63,6.76$ & 0.250 \\
\hline Corticosteroid-dependent disease & 0.80 & $0.33,1.95$ & 0.628 \\
\hline Mayo score $\geq 11$ & 1.05 & $0.22,5.62$ & 0.953 \\
\hline $\mathrm{CAI} \geq 12$ & 0.77 & $0.20,3.01$ & 0.706 \\
\hline C-reactive protein $\leq 0.5 \mathrm{mg} / \mathrm{dl}$ & 2.34 & $0.97,5.83$ & 0.059 \\
\hline Albumin $\geq 3.8 \mathrm{~g} / \mathrm{dl}$ & 1.03 & $0.40,2.61$ & 0.945 \\
\hline \multicolumn{4}{|l|}{ At week 2} \\
\hline $\mathrm{CAI} \leq 4$ & 5.97 & $2.30,16.86$ & $<0.001$ \\
\hline $\mathrm{TL}^{\mathrm{c}} \geq 20.7 \mu \mathrm{g} / \mathrm{ml}$ & 2.75 & $1.13,6.93$ & 0.025 \\
\hline $\mathrm{TL} / \mathrm{CAI} \geq 3.6$ & 5.37 & $2.12,14.50$ & $<0.001$ \\
\hline
\end{tabular}

${ }^{a}$ CAI, clinical activity index; ${ }^{b} \mathrm{CI}$, confidence interval; ${ }^{\mathrm{c}} \mathrm{TL}$, serum infliximab trough level

Logistic regression analysis

Stratified analyses by age, body weight, first TL, and first TL/CAI using median values as cutoffs. The cutoff values of other end points were their reference values low. In clinical practice, there are some acutely ill patients who do not improve initially but who then respond to dose escalation or acceleration compared to standard dosing. Consistent with this, a recent report demonstrated that an accelerated induction regimen improved the colectomy rate in acute severe UC [25]. Immediate switching to other induction options such as tacrolimus or vedolizumab might also be alternatives in patients with low TL or low TL/CAI who are predicted to be non-responders. Our results suggest that the first TL or TL/CAI may be a useful objective marker leading to an individualized induction regimen for acute severe UC. However, further prospective clinical studies are needed to elucidate the advantage of the troughoriented induction strategy in acutely ill patients based on measurement of the first TL.

Development of ATI alters the pharmacokinetics of IFX [26] and is recognized as an important cause of loss of response in patients treated with maintenance or episodic IFX [27]. However, our method measures ATI values only when IFX is absent in sera [17], although we measured ATI at weeks 0,14 , and 30 . No patients were positive for ATI at week 14 in our study, while $67 \%$ of patients with rheumatoid arthritis have detectable ATI within 14 weeks after IFX initiation by the new and sensitive assay [28]. The possibility that the absence of ATI-positive patients was because of technical limitations cannot be excluded, however, the role of ATI in the primary non-responder is still unclear. Clearance of IFX is also influenced by other factors such as sex, body mass index, concomitant use of immunomodulators, disease type, albumin, and inflammatory burden $[18,26]$.

In this multicenter randomized controlled trial, we confirmed that the efficacy of IFX was comparable to that reported in previous trials, including ACT 1 and 2 [8]. This is the first placebo-controlled study with a sufficient sample size from Asia and suggests that IFX is as effective for Asian UC patients as it is for Western populations. Nevertheless, a number of differences between Western and Asian IBD patients are known, including the absence of NOD2 mutant $C D$ and a different gender ratio in $\mathrm{UC}$ 
among Asians [29]. Therefore, it is still important to confirm the reproducibility of clinical trials in the Asian population.

In summary, IFX was confirmed to be effective and safe as therapy for UC. In addition, this study is the first to suggest that the first TL can be used to predict the future treatment outcome. Furthermore, the first TL/CAI was an independent predictor of primary response and was associated with subsequent MH. Our findings may provide critical knowledge for decision making in treatment strategies for acute severe UC. Further prospective studies will be needed to examine the usefulness of the first TLguided induction dosing strategy in acute severe UC.

Acknowledgments This phase 3 randomized controlled study was designed, conducted, and funded by Mitsubishi Tanabe Pharma Corp. The authors thank the patients, investigators, and study personnel who made the trial possible. We also thank the following investigators for their involvement: Akira Nakahara, University of Tsukuba Hospital; Atsushi Yoshida, Ofuna Chuo Hospital; Ayao Torii, Hoshigaoka Medical Center; Hideyuki Hiraishi, Dokkyo Medical University; Hiroaki Takeda, Yamagata University School of Medicine; Hirokazu Oshimoto, Isesaki Municipal Hospital; Hiroshi Yamamoto, Kurashiki Central Hospital; Ichiro Hirata, Tanimukai Hospital; Jun Kato, Okayama University Hospital; Jun Mimura, Nishikobe Medical Center; Junji Shiode, Okayama Saiseikai General Hospital; Katsuhiko Nakai, Matsuda Hospital; Kazuyoshi Yamashita, Japanese Red Cross Sendai Hospital; Keiichi Mitsuyama, Kurume University Hospital; Keiko Shiratori, Tokyo Women's Medical University Hospital; Keisho Kataoka, Kyoto Prefectural University of Medicine; Kenichi Tarumi, Chikuba Hospital for Proctological and Gastrointestinal Diseases; Kenta Motegi, Gunma Prefectural Cancer Center; Kiyotaka Fujise, The Jikei University Kashiwa Hospital; Koji Yakabi, Saitama Medical Center, Saitama Medical University; Kotaro Tashiro, Nanpuh Hospital; Kunihiko Aoyagi, Fukuoka University Hospital; Masaaki Matsukawa, Showa University Koto Toyosu Hospital; Masafumi Nomura, Teine Keijinkai Clinic; Masahiro Iizuka, Akita Red Cross Hospital; Masanori Murakami, Hokkaido P.W.F.A.C. Asahikawa-Kosei Hospital; Masao Saito, Kaisei Hospital; Mineo Kudo, Sapporo Hokuyu Hospital; Mutsuhiro Ikuma, Hamamatsu University Hospital; Nobuhide Oshitani, Osaka City University Hospital; Nobuyuki Chida, National Hospital Organization Sendai Medical Center; Ryota Hokari, National Defense Medical College; Shinichi Okamura, Gunma University Hospital; Shinji Kumagai, Tohoku KoseiNenkin Hospital; Shinji Tanaka, Hiroshima University Hospital; Shozo Okamura, Toyohashi Municipal Hospital; Shunichi Fukao, Nakano GI hospital; Shunji Ishihara, Shimane University Hospital; Susumu Ito, Tokushima University Hospital; Syuji Inoue, National Hospital Organization Kochi National Hospital; Tadashi Yokoyama, Yokoyama Hospital for Gastroenterological Diseases; Takanori Maruo, Japanese Red Cross Osaka Hospital; Takashi Joh, Department of Gastroenterology and Metabolism Nagoya City University Graduate School of Medical Sciences; Takashi Yamaguchi, National Hospital Organization Mito Medical Center; Takayuki Matsumoto, Hyogo College of Medicine; Takayuki Matsumoto, Kyushu University Hospital; Takeshi Nihei, Mito Saiseikai General Hospital; Tatsuo Ogihara, Juntendo University Hospital; Teppei Kabemura, Fukuoka City Medical Association Hospital; Tetsuya Ishida, Japanese Red Cross Oita Hospital; Tomoe Katsumata, Kitasato University East Hospital; Toshifumi Ashida, Asahikawa Medical University Hospital; Toshihide Maruyama, Juntendo University Urayasu Hospital; Toshiro Sugiyama, Toyama University
Hospital; Tsutomu Chiba, Kyoto University Graduate School; Yoshihide Fujiyama, Shiga University of Medical Science; Yusuke Okuyama, Japanese Red Cross Kyoto Daiichi Hospital; Yutaka Endou, Showa University Fujigaoka Hospital.

Conflict of interest Yasuo Suzuki received lecture fees from AbbVie, Mitsubishi Tanabe Pharma, and Zeria Pharmaceutical. Satoshi Motoya has received or is pending receipt of grant support from Ajinomoto Pharmaceuticals and Janssen Pharmaceutical KK and has received lecture fees from Mitsubishi Tanabe Pharma. Haruhiko Ogata received a research grant from Mitsubishi Tanabe Pharma. Hiroaki Ito has received consulting fees from Zeria Pharmaceutical and received lecture fees from Mitsubishi Tanabe Pharma. Noriko Sato and Kunihiko Ozaki are employees of Mitsubishi Tanabe Pharma. Mamoru Watanabe received a research grant from AbbVie GK, Ajinomoto Pharamaceuticals, Eisai, Kyorin Pharmaceutical, Kyowa Hakko Kirin, Mitsubishi Tanabe Pharma, Otsuka Pharmaceutical, Takeda Pharmaceutical, UCB Japan, and Zeria Pharmaceutical. Toshifumi Hibi received a research grant and lecture fees from Abbive GK, Eizai, JIMRO, Mitsubishi Tanabe Pharma, and Zeria Pharmaceutical. The other authors have no conflicts of interest.

Open Access This article is distributed under the terms of the Creative Commons Attribution-NonCommercial 4.0 International License (http://creativecommons.org/licenses/by-nc/4.0/), which permits any noncommercial use, distribution, and reproduction in any medium, provided you give appropriate credit to the original author(s) and the source, provide a link to the Creative Commons license, and indicate if changes were made.

\section{References}

1. Podolsky DK. Inflammatory bowel disease. $\mathrm{N}$ Engl $\mathrm{J}$ Med. 2002;347:417-29.

2. Goldsmith JR, Sartor RB. The role of diet on intestinal microbiota metabolism: downstream impacts on host immune function and health, and therapeutic implications. J Gastroenterol. 2014;49:785-98.

3. Sheehan D, Moran C, Shanahan F. The microbiota in inflammatory bowel disease. J Gastroenterol. 2015;50:495-507.

4. Breese EJ, Michie CA, Nicholls SW, et al. Tumor necrosis factor $\alpha$-producing cells in the intestinal mucosa of children with inflammatory bowel disease. Gastroenterology. 1994;106: 1455-66.

5. Plevy SE, Landers CJ, Prehn J, et al. A role for TNF- $\alpha$ and mucosal T helper-1 cytokines in the pathogenesis of Crohn's disease. J Immunol. 1997;159:6276-82.

6. Targan SR, Hanauer SB, van Deventer SJ, et al. A short-term study of chimeric monoclonal antibody cA2 to tumor necrosis factor $\alpha$ for Crohn's disease. N Engl J Med. 1997;337:1029-35.

7. Hanauer SB, Feagan BG, Lichtenstein GR, et al. Maintenance infliximab for Crohn's disease: the ACCENT I randomised trial. Lancet. 2002;359:1541-9.

8. Rutgeerts P, Sandborn WJ, Feagan BG, et al. Infliximab for induction and maintenance therapy for ulcerative colitis. $\mathrm{N}$ Engl $\mathrm{J}$ Med. 2005;353:2462-76.

9. Faubion WA Jr, Loftus EV Jr, Harmsen WS, et al. The natural history of corticosteroid therapy for inflammatory bowel disease: a population-based study. Gastroenterology. 2001;121:255-60.

10. Mehta SJ, Silver AR, Lindsay JO. Review article: strategies for the management of chronic unremitting ulcerative colitis. Aliment Pharmacol Ther. 2013;38:77-97. 
11. Mowat C, Cole A, Windsor A, et al. Guidelines for the management of inflammatory bowel disease in adults. Gut. 2011; 60:571-607.

12. Laharie D, Bourreille A, Branche J, et al. Ciclosporin versus infliximab in patients with severe ulcerative colitis refractory to intravenous steroids: a parallel, open-label randomised controlled trial. Lancet. 2012;380:1909-15.

13. Afif W, Loftus EV Jr, Faubion WA, et al. Clinical utility of measuring infliximab and human anti-chimeric antibody concentrations in patients with inflammatory bowel disease. Am J Gastroenterol. 2010;105:1133-9.

14. Yanai H, Lichtenstein L, Assa A, et al. Levels of drug and antidrug antibodies are associated with outcome of interventions after loss of response to infliximab or adalimumab. Clin Gastroenterol Hepatol. 2015;13:522-30.

15. Schroeder KW, Tremaine WJ, Ilstrup DM. Coated oral 5-aminosalicylic acid therapy for mildly to moderately active ulcerative colitis. A randomized study. N Engl J Med. 1987; 317:1625-9.

16. Lichtiger S, Present DH, Kornbluth A, et al. Cyclosporine in severe ulcerative colitis refractory to steroid therapy. N Engl J Med. 1994;330:1841-5.

17. Maini RN, Breedveld FC, Kalden JR, et al. Therapeutic efficacy of multiple intravenous infusions of anti-tumor necrosis factor $\alpha$ monoclonal antibody combined with low-dose weekly methotrexate in rheumatoid arthritis. Arthritis Rheum. 1998; 41:1552-63.

18. Fasanmade AA, Adedokun OJ, Olson A, et al. Serum albumin concentration: a predictive factor of infliximab pharmacokinetics and clinical response in patients with ulcerative colitis. Int J Clin Pharmacol Ther. 2010;48:297-308.

19. Lee KM, Jeen YT, Cho JY, et al. Efficacy, safety, and predictors of response to infliximab therapy for ulcerative colitis: a Korean multicenter retrospective study. J Gastroenterol Hepatol. 2013; 28:1829-33.
20. Zampeli E, Gizis M, Siakavellas SI, et al. Predictors of response to anti-tumor necrosis factor therapy in ulcerative colitis. World J Gastrointest Pathophysiol. 2014;5:293-303.

21. Armuzzi A, Pugliese D, Danese S, et al. Infliximab in steroiddependent ulcerative colitis: effectiveness and predictors of clinical and endoscopic remission. Inflamm Bowel Dis. 2013;19:1065-72.

22. Adedokun OJ, Sandborn WJ, Feagan BG, et al. Association between serum concentration of infliximab and efficacy in adult patients with ulcerative colitis. Gastroenterology. 2014;147:1296-307.

23. Seow $\mathrm{CH}$, Newman A, Irwin SP, et al. Trough serum infliximab: a predictive factor of clinical outcome for infliximab treatment in acute ulcerative colitis. Gut. 2010;59:49-54.

24. Colombel JF, Rutgeerts P, Reinisch W, et al. Early mucosal healing with infliximab is associated with improved long-term clinical outcomes in ulcerative colitis. Gastroenterology. 2011;141:1194-201.

25. Gibson DJ, Heetun ZS, Redmond CE, et al. An accelerated infliximab induction regimen reduces the need for early colectomy in patients with acute severe ulcerative colitis. Clin Gastroenterol Hepatol. 2015;13:330-5.

26. Ordás I, Mould DR, Feagan BG, et al. Anti-TNF monoclonal antibodies in inflammatory bowel disease: pharmacokineticsbased dosing paradigms. Clin Pharmacol Ther. 2012;91:635-46.

27. Lee LY, Sanderson JD, Irving PM. Anti-infliximab antibodies in inflammatory bowel disease: prevalence, infusion reactions, immunosuppression and response, a meta-analysis. Eur J Gastroenterol Hepatol. 2012;24:1078-85.

28. Krintel SB, Grunert VP, Hetland ML, et al. The frequency of anti-infliximab antibodies in patients with rheumatoid arthritis treated in routine care and the associations with adverse drug reactions and treatment failure. Rheumatology. 2013;52:1245-53.

29. Inoue N, Tamura K, Kinouchi Y, et al. Lack of common NOD2 variants in Japanese patients with Crohn's disease. Gastroenterology. 2002;123:86-91. 